\title{
Design and Implementation of Hybrid Light and Microwave Switches Based on Wavelength Selective Switch for Future Satellite Networks
}

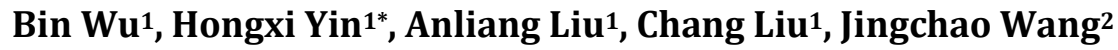 \\ ${ }^{1}$ Laboratory of Optical Communications and Photonic Technology, School of Information and Communication \\ Engineering, Dalian University of Technology, Dalian, China \\ ${ }^{2}$ Institute of China Electronic System Engineering Company, Beijing, China \\ Email: "hxyin@dlut.edu.cn
}

Received 3 November 2015; accepted 26 February 2016; published 2 March 2016

\begin{abstract}
A hybrid switching node structure with light and microwave links is proposed, which is applicable to the future data relay satellite systems, aiming at the development trend of coexistence of lightlink and microwave-link in the future. An experimental system for the light and microwave hybrid switching node based on wavelength selective optical switches (WSS) and optical transceiver modules, is established. It is shown by our experiment that this hybrid switching node can realize the dynamic bandwidth allocation and wavelength routing while the bit error rate of light link is less than $10^{-12}$, which provides a method for solving the hybrid switching problem of light-link and microwave-link on the future data relay satellite systems.
\end{abstract}

\section{Keywords}

Light-Link and Microwave-Link, Data Relay Satellite, WSS, Hybrid Switching

\section{Introduction}

With the continuous developments of high-speed data communication, navigation and positioning, high resolution image acquisition and deep space exploration, the transmission demand of large capacity and high-speed data relay satellite services for inter-satellites is rapidly increasing. Therefore, the inter-satellite light-link has become an important development trend for data relay satellites. In recent years, many developed countries, such as Europe, America and Japan, have successively carried out theoretical research and spaceborne demonstration about the data relay satellite system with inter-satellite light-links, and launched out into corresponding engineering verification and satellite networking project [1] [2]. With the improving performance of inter-satellite light link, it has been a trend for satellite communication (SATCOM) to construct an satellite optical networks based on light link and achieve broadband satellite data communication, which is a principal way to solve 
contradiction between the exponentially increasing information and the limited bandwidth in existing satellite communication. However, the matured satellite largely depends on microwave link for now, because communication based on microwave can realize multi-beam coverage and get the merits of widespread applications, accessing rapidly and high degree of technological maturity. Moreover, microwave link in space-terrestrial communication system can partially overcome the influence of atmospheric turbulence to ensure the reliability in the satellite communication link. Therefore, microwave link will still be the main body of satellite communication system and space information network system in the future.

The inter-satellite light link possesses many advantages such as high bandwidth, small size, low power consumption, excellent transmission performance and etc., while microwave link in satellite-ground communication could make full use of the mature technology of ground station and avoid the severe influence of atmospheric turbulence on the light link. Hence, the satellite information system with high bandwidth and flexibility will definitely be a heterogeneous hybrid network in the future where light-link and microwave-link can achieve coexisting and complementary advantages. When the information from different satellites or ground stations converges to a data relay satellite node, there will be a spaceborne convergence of microwave link and light link. In this situation, data relay satellite node should have the ability to switch the data of backbone light link as well as to accomplish data access from light-link and microwave-link to build a bridge for both light and microwave network in the inter-satellite and space-terrestrial communications. In recent years, with the continuous development of satellite light communication and the trend of multi-satellite networking in many countries, to solve the problem of light and microwave hybrid switching on data relay satellite is becoming increasingly urgent.

In this paper, a structure of spaceborne light-link and microwave-link hybrid switch is proposed based on wavelength selective optical switch (WSS). A hybrid switching system is designed and experimentally implemented, which can achieve dynamical reconfiguration, optical cross connection and add/drop multiplexing. It is shown by the experimental result of light and microwave hybrid switching that the proposed hybrid switching system can be available for the future satellite networks.

\section{Structure for Spaceborne Light-Link and Microwave-Link Hybrid Node}

The data relay satellite system based on light and microwave link hybrid switching is shown in Figure 1. The satellite backbone network is formed between Geosynchronous Satellites (GSO) by the light link, which takes full advantage of high speed, large capacity, high bandwidth, low power consumption, small volume and anti-electromagnetic interference. Backbone network and access network are connected with light-links or microwave-links, while satellite and ground station are mainly connected by relative mature microwave links [3]. Therefore, the data relay node in backbone network is a hybrid satellite node, which should have the ability of both microwave access and optical switching.

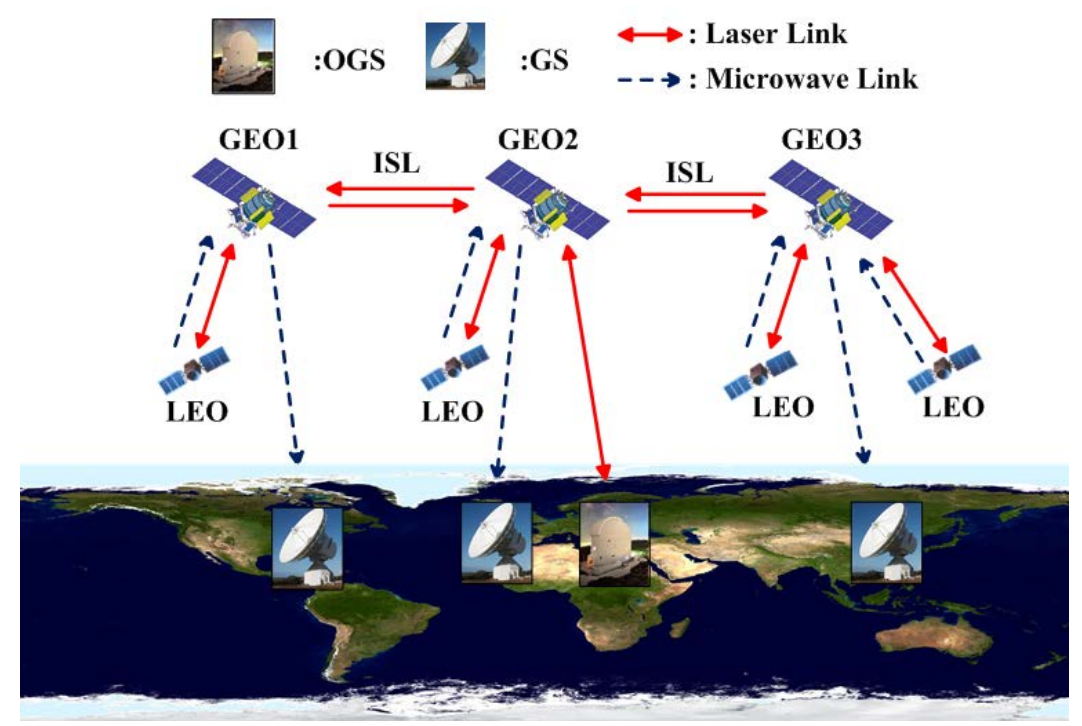

Figure 1. Data relay satellite system with hybrid light-links and microwave-links. 
The structure of spaceborne light and microwave hybrid switches based on WSS is shown in Figure 2. It can interconnect light links between the backbone network, realize optical cross-connect and wavelength routing and simultaneously access light and microwave signals. The accessing microwave signal from low earth orbit (LEO) satellites or ground station (GS) is modulated into optical domain to implement transparent processing of microwave signal and then it passes the switching matrix before sent to the next satellite node. The hybrid node also has the ability of optical cross connection and add-drop multiplexing [4] [5].

The switching fabric is made up of $1 \times \mathrm{N}$ wavelength selective switching matrix, shown in Figure 3 . WSS has the ability to independently switch any wavelength to any port without any restrictions imposed by the switching of other wavelengths. WSS is completely reconfigurable by allocating channel spacing and bandwidth dynamically. This property improves the spectrum efficiency and makes it applicable to dynamically changeable network with limited spectrum resources [6] [7].

\section{Experimental Results of Hybrid Switching Node}

The test system of light and microwave hybrid switching node is connected as shown in Figure 4, which includes the Agilent E4438 vector signal generator, the DWDM optical transceiver, the optical modulator, the polarization controller, the wavelength division multiplexer and de-multiplexer, the $1 \times 4$ wavelength selective switch, the photodetector, the Anritsu MS9740A optical spectrum analyzer, the Agilent N9020A MXA signal analyzer and the Anritsu MP1800A BERT. The operating wavelength of the optical transmitter module is $1554.94 \mathrm{~nm}$ and $1553.33 \mathrm{~nm}$ respectively, which corresponds to Channel 28 and Channel 30 in dense wavelength division multiplexing standard (ITU-T). The insertion loss of WSS is approximately $7 \mathrm{~dB}$, and the isolation is higher than $34 \mathrm{~dB}$.

Firstly, the channel spacing and bandwidth allocation for $1 \times 4$ WSS will be tested. The wide-spectrum light from a light diode enters the input "com" port of the WSS through a variable optical attenuator. A personal computer (PC) is employed to send control commands through its serial port and after the channel spacing, wavelength routing and attenuation for each channel are allocated the spectra is observed by optical spectrum analyzer. The bandwidths assigned to each channel are $25 \mathrm{GHz}, 50 \mathrm{GHz}, 100 \mathrm{GHz}$ and $200 \mathrm{GHz}$, respectively and the attenuation for each channel is $0 \mathrm{~dB}$. As shown in Figure 5, the $-3 \mathrm{~dB}$ bandwidths of the four channels are

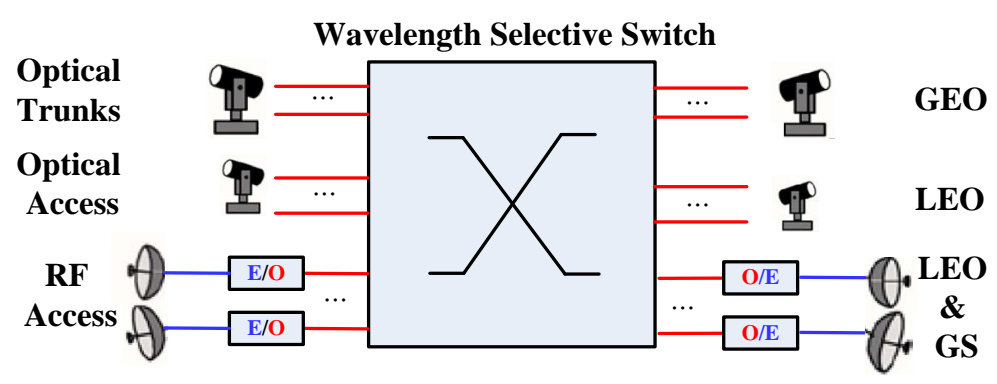

Figure 2. Light and microwave hybrid switching node for data relay satellite.

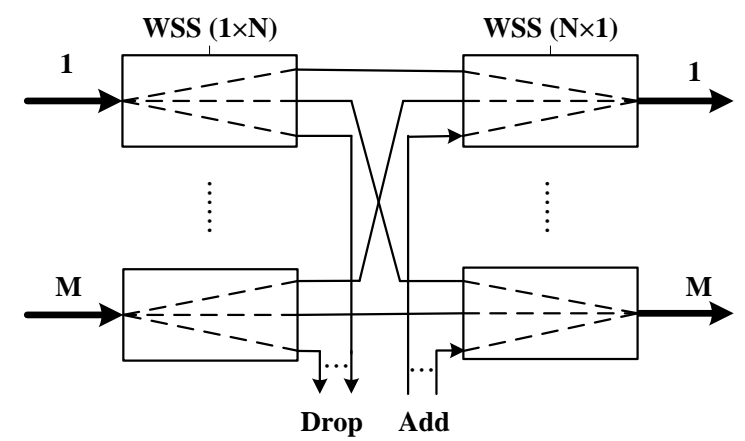

Figure 3. Schematic of the internal structure of the wavelength selective switching matrix. 
$0.19 \mathrm{~nm}, 0.39 \mathrm{~nm}, 0.78 \mathrm{~nm}$ and $1.59 \mathrm{~nm}$, respectively.

Secondly, let's verify the hybrid switching ability between light-links and microwave-links. The experiment is based on the designed testbed shown in Figure 4. The microwave link signal is generated by E4438 vector signal generator. The rate of the digital baseband signal is $50 \mathrm{Mbps}$ and the carrier frequency is $5 \mathrm{GHz}$, and the modulation mode is 16QAM. The microwave signal is transformed into an optical signal and then, it passes through the hybrid node $(\mathrm{B} \rightarrow \mathrm{D})$ and outputs from the third port of the wavelength division demultiplexer at the bottom right. Its spectra are shown in Figure 6 and Figure 6(a) shows the spectrum of input 5 GHz signal modulated by 50 Mbps RF signal, and Figure 6(b) displays the RF signal spectrum of hybrid light-microwave switching node. It can be seen by comparison of two figures that the central frequency of this signal is $5 \mathrm{GHz}$ and the total loss of the switching system is $29 \mathrm{~dB}$. The light link data is generated by the MP1800A BERT and an optical transmitter. The test pattern is NRZ $2^{31}-1$ of $10 \mathrm{Gbps}$ data rate. The signal outputs from the first port of the wavelength division demultiplexer through the hybrid node $(A \rightarrow C)$ and the bit error rate of light link in the loopback test is less than $10^{-12}$.

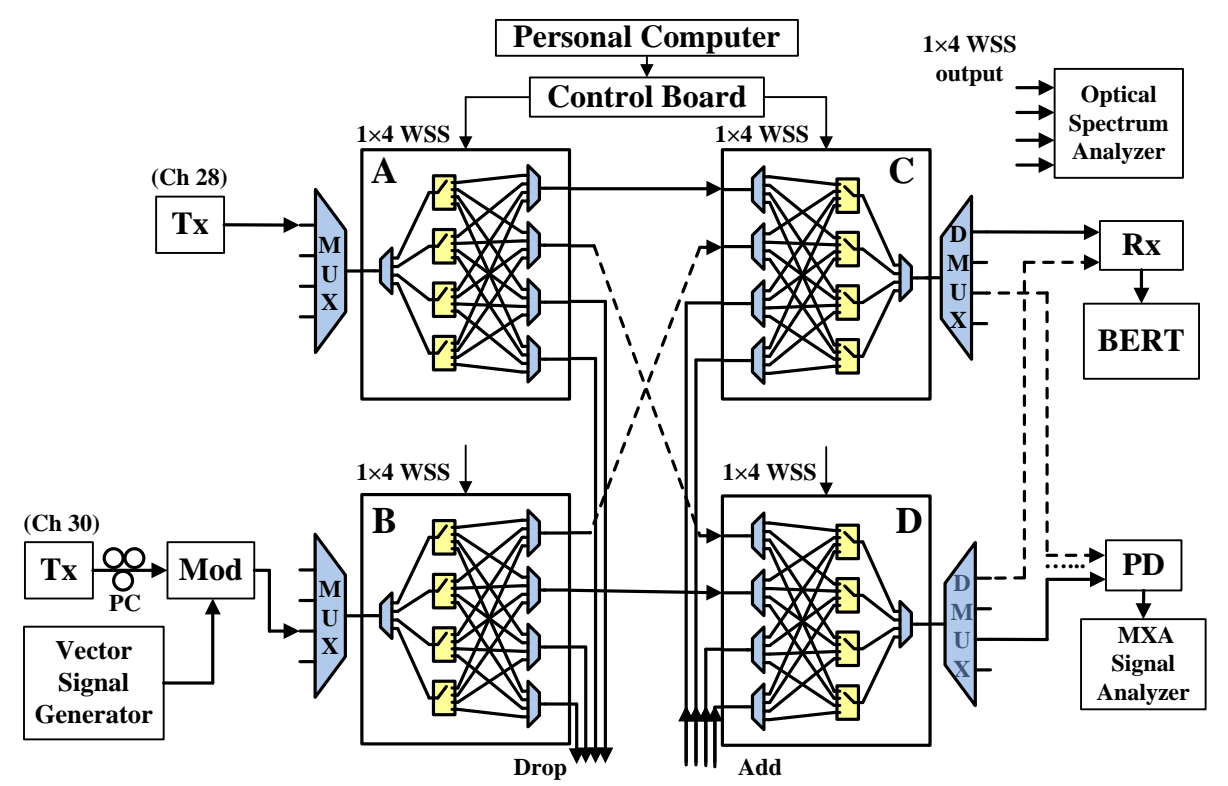

Figure 4. Experimental system for the light and microwave hybrid switching node.

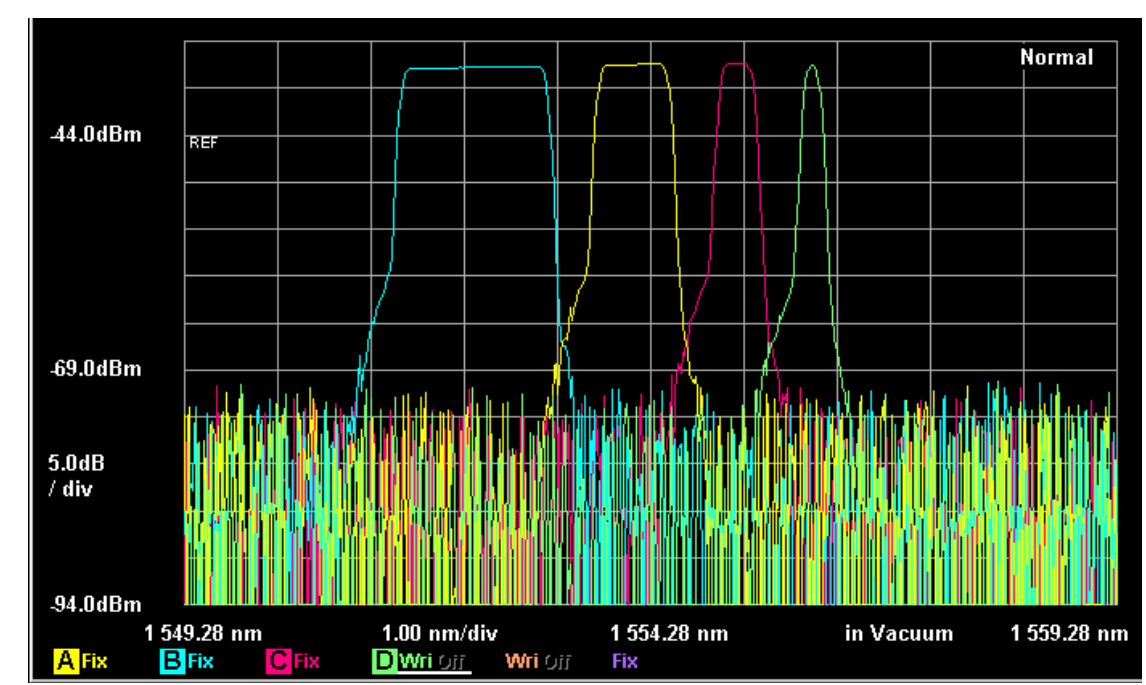

Figure 5. Spectra of the four channels (the SLICE number assigned to each channel is 16, 8, 4, 2 respectively). 

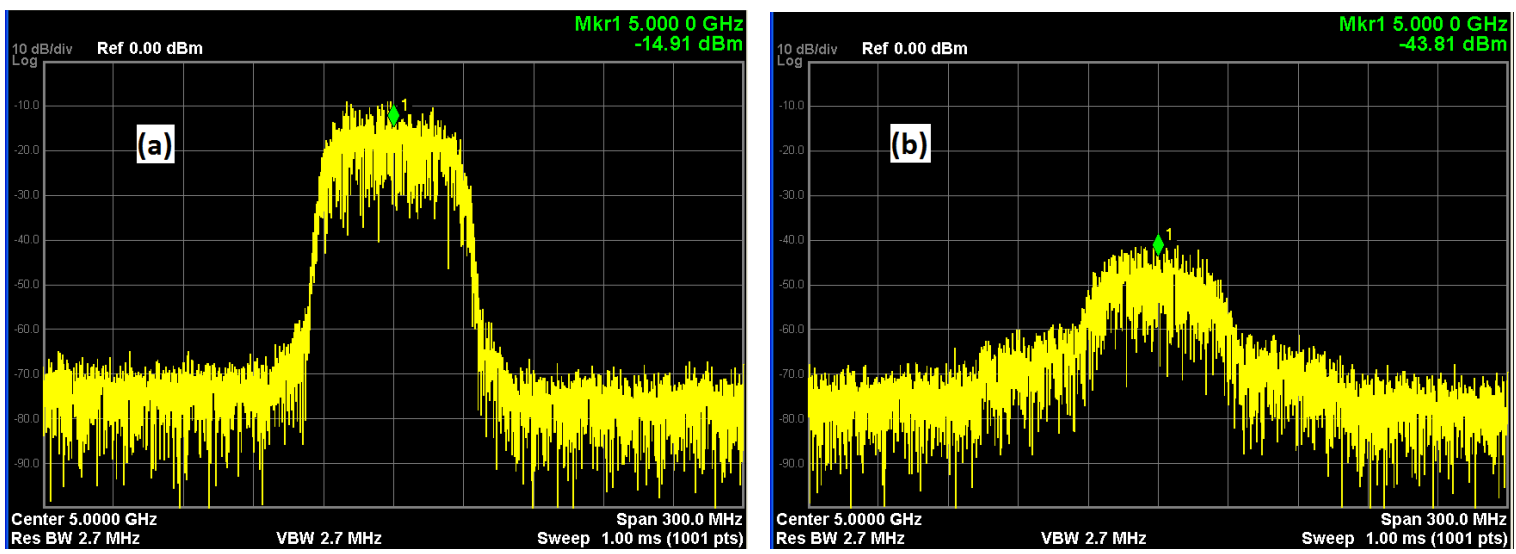

Figure 6. Spectra of the input and output $5 \mathrm{GHz}$ signals modulated by $50 \mathrm{Mbps}$ RF signal. (a) Input RF signal after 16QAM modulation; (b) Output RF signal of light and microwave hybrid switching node.

The dotted line shown in Figure 4 indicates the signal flow of between the light-links and microwave-links after switching controlled by the computer $(\mathrm{A} \rightarrow \mathrm{D}, \mathrm{B} \rightarrow \mathrm{C})$. The experimental results are consistent with those of previous tests. The hybrid switching node designed can accomplish data exchange between light-links and microwave-links to achieve the function of the data relay satellite.

\section{Conclusion}

In this paper, a light-microwave hybrid switching node applicable to data relay satellites, is designed and an experimental platform based on $1 \times 4$ WSS and optical transceiver module, is set up. The experiment results indicate that the hybrid node can realize dynamic bandwidth allocation and wavelength routing, which highly improves the spectrum efficiency of spaceborne communication system. It reveals through experimental testing that this node can implement the light and microwave hybrid switching. The signal of microwave link is transformed into optical domain to implement the transparent processing using optical transceiver module, while the bit error rate of light link in the loopback test is less than $10^{-12}$. The hybrid node designed is applicable for the situation of converge for light-links and microwave-links on a data relay satellite, which has many advantages such as high spectrum efficiency, transparent data processing and etc.

\section{Acknowledgements}

This work was supported in part by the National Natural Science Foundation of China (NSFC) under Grants 61231011 and 61071123, and The First HAEPC Science and Technology Project in 2015(5217Q014006U).

\section{References}

[1] Arruego, I., Guerrero, H., Rodrıguez, S., Martınez-Oter, J., Jimenez, J.J., et al. (2009) OWLS: A Ten-Year History in Optical Wireless Links for Intra-Satellite Communications. IEEE Journal on Selected Areas in Communications, 27, 1599-1610. http://dx.doi.org/10.1109/JSAC.2009.091210

[2] Hanada, T., Yamakawa, S. and Kohata, H. (2011) Study of Optical Inter-orbit Communication Technology for Next Generation Space Data-relay Satellite. Proceedings of Free-Space Laser Communication Technologies Conference of SPIE, San Francisco, 26-27 January 2011, 7923.

[3] Hossein, S. and Murat, U. (2013) End-to-End Performance of Mixed RF/FSO Transmission Systems. Journal of Optical Communications and Networking, 5, 1139-1144. http://dx.doi.org/10.1364/JOCN.5.001139

[4] Daneshmand, M. and Mansour, R.R. (2011) RF MEMS Satellite Switch Matrices. Microwave Magazine, 12, 92-109. http://dx.doi.org/10.1109/MMM.2011.941417

[5] Hwan, S.C., Sun, H.C., Sang, S.L. and Kwangjoon, K. (2008) Experimental Demonstration of Optical Multicast Using WSS Based Multi-Degree ROADM. Proceedings of Optical Fiber Communication Conference and Exposition (OFC/NFOEC) and the National Fiber Optic Engineers Conference, San Diego, 24-28 February 2008, 88-91.

[6] Winston, I.W., Philip, N.J. and Ankitkumar, N.P. (2013) Wavelength Contention-Free via Optical Bypass within a 
Colorless and Directionless ROADM [Invited]. Journal of Optical Communications and Networking, 5, 220-229. http://dx.doi.org/10.1364/JOCN.5.00A220

[7] Strasser, T.A. and Wagener, J.L. (2010) Wavelength-Selective Switches for ROADM Applications [Invited]. IEEE Journal of Selected Topics in Quantum Electronics, 16, 1150-1157. http://dx.doi.org/10.1109/JSTQE.2010.2049345 